\title{
Percepção dos alunos acerca das estratégias de promoção do sucesso educativo e envolvimento com a escola
}

\author{
Relation between school success promotion \\ strategies and student's engagement \\ with school
}

\author{
Adelaide DIAS \\ João Tiago OLIVEIRA \\ Paulo Alexandre Soares MOREIRA ${ }^{1}$ \\ Leonel ROCHA ${ }^{2}$
}

\begin{abstract}
Resumo
As associações entre as percepções dos alunos acerca das características da escola e o envolvimento dos alunos com a escola são ainda pouco compreendidas. O objetivo deste estudo foi avaliar a relação entre a percepção dos alunos acerca das estratégias de promoção de sucesso educativo e o seu envolvimento com a escola. Participaram neste estudo 529 alunos do $2^{\circ}$ e $3^{\circ}$ ciclo de um município do Norte de Portugal, Vila Nova de Famalicão. Os resultados demonstram a existência de uma correlação positiva e estatisticamente significativa entre a percepção dos alunos acerca das estratégias de otimização de aprendizagem e o envolvimento escolar. Estes resultados têm fortes implicações para os esforços de melhoria e da eficácia da escola, incluindo para a promoção de trajetórias acadêmicas positivas.
\end{abstract}

Palavras-chave: Educação; Percepção; Realização; Rendimento escolar.

\begin{abstract}
The association between the students' perception of the school characteristics and the students' engagement with school requires further research. The aim of the study was to describe the association between the students' perception of school success promotion strategies and students' engagement with school. The sample consisted of 529 students, enrolled in elementary and middle-school classes, from a municipality in Northern Portugal, Vila Nova de Famalicão. The results showed a statistically significant positive correlation between the students' perception of the school success promoting strategies and students' engagement with school. These results have strong implications with respect to the efforts to improve education and school effectiveness, including the promotion of positive academic trajectories.
\end{abstract}

Keywords: Education; Perception; Achievement; Academic achievement.

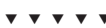

1 Universidade Lusíada do Porto, Observatório da Melhoria e Eficácia da Escola. R. Doutor Lopo de Carvalho, 4369-006, Porto, Portugal. Correspondência para/Correspondence to: P.A.S. MOREIRA. E-mail: <paulomoreira@por.ulusiada.pt>.

2 Vereador. Vila Nova de Famalicão, Portugal. 
As pesquisas têm reconhecido a existência de uma relação próxima entre os níveis de educação de um país e o seu desenvolvimento econômico e social (United Nations Educational Scientific and Cultural Organization [Unesco], 2005; 2008; Vare \& Scott, 2007).

O principal desafio que se coloca às sociedades atuais é garantir que todos tenham acesso a uma educação de qualidade, equitativa e inclusiva, o que permitirá o melhor funcionamento do país, desenvolvendo e otimizando as suas competências (Ainscow, 2010; McKeown, 2002). Dessa forma, é uma questão de interesse público que os sistemas educativos não só promovam que os conteúdos curriculares sejam transmitidos de forma desejável, como também fomentem os fatores que potenciam o desenvolvimento do aluno em sua trajetória acadêmica, incluindo o nível de seu envolvimento com a escola.

Resultante das evidências de que vários indicadores determinantes das trajetórias acadêmicas são passíveis de serem melhorados por meio de estratégias intencionais desenvolvidas pelas escolas, tem-se assistido nos últimos anos a uma crescente importância atribuída a esses esforços para aumentar a eficácia educativa. O International Congress of School Improvement and School Efficacy é um exemplo de um organismo internacional de promoção da eficácia educativa, pela via da promoção de estratégias nesse sentido.

Na organização escolar, existem evidências de que a promoção de determinadas dimensões tem um impacto significativo positivo nas trajetórias acadêmicas dos alunos (Epstein \& Sheldon, 2002; Lee \& Burkam, 2003), principalmente quanto a: (a) plasticidade do sistema escolar: capacidade da escola e do contexto educativo de se adaptar às características e necessidades dos alunos (Bryk, Easton, Kerbeow, Rollow, \& Sebring, 1994; Reynolds, A. Harris, Clarke, Harris, \& James, 2006; Taylor \& Adelman, 1999); (b) monitorização constante (Gullatt \& Lemoine, 1997; Taylor \& Adelman, 1999; Wagstaff, Combs, \& Jarvis, 2000); (c) existência de atividades extracurriculares (DeKalb, 1999; Feldman \& Matjasko, 2005; Gilman, Meyers, \& Perez, 2004);

188 (d) tutoria: desenvolvida por professores ou colegas mais velhos que apoiam os alunos com maiores dificuldades (Gonzales, Richards, \& Seeley, 2002; Tierney, Grossman, \& Resch, 1995); (e) promoção de atividades de aprendizagem ativa: os alunos realizam suas tarefas de modo autônomo e com os seus pares (Bell \& Kozlowski, 2008; Taraban, Box, Myers, Pollard, \& Bowen, 2007; Yoder \& Hochevar, 2005); (f) aprendizagem individualizada: os professores adaptam suas práticas de ensino a cada aluno (Adelman \& Taylor, 1993; W. Stainback \& Stainback, 1992; Switzer, 2004); (g) utilização de tecnologias educativas, em casa e na escola (Hartley, 2007; Imel, 1999; Wesley, 2004); e (h) educação para a carreira (Stone, 2004; Stone \& Aliaga, 2002).

Por outro lado, tem-se mostrado eficaz a atitude sistemática das escolas em monitorizar os esforços de melhoria, por meio de mecanismos de autoavaliação (Creemers \& Reezigt, 2005; Sun, Creemers, \& de Jong, 2007), o que favorece a persecução daqueles objetivos (Hofman, Dijkstra, \& Hofman, 2005; Schildkamp, Visscher, \& Luyten, 2009).

As trajetórias acadêmicas são fenômenos multifatoriais. Concretamente, o rendimento acadêmico resulta de dinâmicas não lineares entre as dimensões do aluno, da família, da escola e da comunidade (Moreira, Dias, F. M. Vaz, \& Vaz, 2013). $O$ envolvimento do aluno é um construto referente às organizações psicológicas implicadas nas significações da escola, e é o principal preditor quer do rendimento, quer do abandono escolar precoce, bem como de outros indicadores positivos e negativos da trajetória acadêmica (Ferreira, Santos, Fonseca, \& Haase, 2007; Henry, Knight, \& Thornberry, 2012) e de situações de risco (Motti-Stefanidi \& Masten, 2013; Totura, Karver, \& Gesten, 2013). Além disso, o envolvimento dos alunos com a escola é um preditor significativo do estatuto educacional e ocupacional na idade adulta (Abbott Chapman et al., 2013).

O envolvimento escolar é um construto emergente, constituindo um dos principais construtos integradores das diferentes dimensões da experiência dos alunos em relação à escola. Existem várias abordagens sobre o envolvimento dos alunos com a escola, todas coincidentes com a premissa 
de que o envolvimento dos alunos é multidimensional (Fredricks et al., 2011). Nesse sentido, Appleton, Christenson, Kim e Reschly (2006) conceptualizam o envolvimento escolar no nível das dimensões cognitiva, psicológica, comportamental e acadêmica.

Recentemente, tem aumentado o consenso acerca da necessidade de compreender as interações entre a dimensão contextual e as bases psicobiológicas do comportamento, também no domínio das trajetórias acadêmicas. Apesar de o envolvimento dos alunos com a escola se referir iminentemente à sua experiência subjetiva, esta é influenciada de forma significativa por dimensões contextuais (Moreira, Dias, Vaz, Rocha, \& Freitas, 2012c). De fato, enquanto conceito organizador das dimensões dos modelos motivacionais e de persistência educacional, o envolvimento dos alunos com a escola reflete as mútuas influências das dimensões individuais e contextuais na construção de significados (Moreira et al., 2013). Por exemplo, a dimensão cognitiva do envolvimento com a escola tende a refletir a influência dos contextos desenvolvimentais e relacionais em que o aluno se move. O envolvimento dos pais com a escola e a valorização dela para o futuro exercem influência significativa na construção das percepções do aluno sobre a relevância da escola para seu futuro (Moreira, Dias, Pettrachi, \& Vaz, 2012b). De forma análoga, o grau médio de envolvimento dos alunos apresenta consistência com as características das respectivas escolas, de que é exemplo o fato de alunos de escolas com história de bons resultados acadêmicos apresentarem maiores níveis de envolvimento do que aqueles oriundos de escolas com histórico de resultados pobres (Moreira et al., 2012a).

A questão acerca das características contextuais que têm impacto no envolvimento dos alunos com a escola é de grande relevância para os sistemas educativos, já que permite a adoção de medidas que favoreçam níveis mais adaptativos de envolvimento, o que por sua vez tem o potencial de promover indicadores adaptativos de trajetórias acadêmicas e de prevenir indicadores negativos. Existem evidências de que características como o envolvimento dos pais com a escola, ou a existência de estratégias de promoção do sucesso educativo, favorecem indicadores positivos de trajetórias acadêmicas.

Apesar disso, a investigação acerca das associações entre os esforços da escola em promover estratégias de promoção do sucesso educativo e o envolvimento dos alunos ela (principalmente o envolvimento cognitivo e emocional) encontra-se numa fase relativamente embrionária. O estudo do impacto dos esforços de melhoria no envolvimento dos alunos com a escola pode ser feito por múltiplas vias, seja por indicadores objetivos (notas, faltas etc.), seja por indicadores da percepção dos estudantes. A linha de investigação que tem sido valorizada é a primeira, sendo que apenas recentemente tem havido alguns desenvolvimentos na segunda. Apesar da importância de se avaliar o impacto das estratégias de promoção do sucesso educativo por meio de indicadores objetivos, é relevante conhecer tal impacto também no nível da percepção dos alunos. Dentre outras razões, pode-se citar: (a) o envolvimento com a escola é iminentemente um fenômeno da experiência subjetiva dos alunos; (b) as percepções são fortes preditores de indicadores mais objetivos, incluindo indicadores comportamentais; e (c) a compreensão da experiência subjetiva e da forma como esta interage com estratégias da escola é de grande relevância para a compreensão do processo (nesse caso, do processo de promover níveis mais desejáveis de envolvimento).

Recentemente, alguns estudos têm demonstrado que as percepções positivas dos alunos acerca dos esforços de melhoria da escola acentuam seu níveis de envolvimento (Cavendish, 2013). Além disso, percepções dos alunos e características da escola interagem de forma recíproca (Van Ryzin, 2011), o que demonstra a grande relevância de se compreender a dinâmica entre características da escola e envolvimento dos alunos.

Um dos principais entraves para se descrever a interação entre as estratégias de promoção do sucesso educativo e o envolvimento dos alunos com a escola é a escassez de instrumentos específicos para avaliar as percepções dos estudantes acerca daqueles esforços. Recentemente foram desen- 
volvidos instrumentos específicos para tanto, com características psicométricas adequadas à natureza hierárquica do fenômeno (percepção dos alunos, dentro de cada escola) (Moreira, Oliveira, Dias, Vaz, \& Torres-Oliveira, 2014).

Apesar da relevância de se compreender a associação entre as percepções dos alunos quanto aos esforços de melhoria e os seus níveis de envolvimento com a escola, não se conhece nenhum estudo que tenha respondido a essa questão com um instrumento de avaliação específico, de forma independente.

Por essa razão, este foi um estudo exploratório. A pesquisa teve como principal objetivo avaliar a relação entre a percepção dos alunos acerca das estratégias de promoção do sucesso educativo e o envolvimento dos mesmos com a escola, no contexto de um Plano Municipal de Melhoria da Escola, desenvolvido de forma intencional e sistemática num município do norte de Portugal, e acompanhado pelo Observatório da Melhoria e da Eficácia da Escola.

\section{Método}

\section{Participantes}

Participaram do estudo 529 alunos de quatro escolas do município de Vila Nova de Famalicão, situado no norte de Portugal. Destes, 216 alunos frequentavam o $2^{\mathrm{a}}$ Ciclo do Ensino Básico $\left(5^{\circ}\right.$ e $6^{\circ}$ anos) e 313 o $3^{\circ}$ Ciclo do Ensino Básico $\left(7^{\circ}, 8^{\circ}\right.$ e $9^{\circ}$ anos). Embora o status socioeconômico não tenha sido controlado na amostra, tendencialmente, 50\% dos alunos que frequentavam as escolas do município recebiam algum tipo de apoio econômico estatal, motivo pelo que este estuda os considera como de nível socioeconômico baixo.

Por serem do mesmo município, as escolas apresentam características sociodemográficas semelhantes entre si, embora variem em dimensão. A escola A era frequentada por 1071 alunos, a escola B por 524, a escola C por 694 e a escola D por 355. Uma das escolas era considerada Território

190 Educativo de Intervenção Prioritária (TEIP). Em
Portugal a escolaridade obrigatória está organizada em quatro níveis de ensino: $1^{\circ}$ Ciclo do Ensino do Básico: $1^{\circ}$ a $4^{\circ}$ ano; $2^{\circ}$ Ciclo do Ensino Básico: $5^{\circ} \mathrm{e}$ $6^{\circ}$ ano; $3^{\circ}$ Ciclo do Ensino Básico: $7^{\circ}$ a $9^{\circ}$ ano; e Ensino Secundário: $10^{\circ}$ a $12^{\circ}$ ano.

Os Territórios Educativos de Intervenção Prioritária foram criados em 1996 pelo Ministério da Educação Português, através do Despacho 147-B/ME/96. Compreendem escolas e agrupamentos de escolas que têm um número elevado de alunos em risco de exclusão social, abandono e insucesso escolar. O programa tem como principal objetivo: "Promover a igualdade no acesso e no sucesso educativos da população escolar em idade de frequência do ensino básico, universal e gratuito, muito em particular das crianças e dos jovens em situação de risco de exclusão (social e escolar)" (Costa, Sousa, \& Neto-Mendes, 2000, p.83).

\section{Instrumentos}

Para avaliação do envolvimento escolar foi utilizada a School Engagement Inventory (Moreira, Vaz, Dias, \& Petracchi, 2009), adaptado para Portugal por um dos autores deste estudo. É uma medida de acesso livre. A escala é constituída por 35 itens com tipo de resposta Likert, 1 = Discordo Totalmente; 2 = Discordo; 3 = Concordo; 4 = Concordo Totalmente. $\mathrm{O}$ instrumento avalia duas dimensões do envolvimento escolar: a cognitiva e a psicológica. A subescala Envolvimento Escolar Cognitivo avalia componentes do envolvimento escolar mais internos, como a autorregulação, a relevância do trabalho escolar para o futuro, os objetivos pessoais e autonomia. É constituída pelas subescalas Controle e Relevância do Trabalho da Escola (e.g., "A maioria do que é importante saber aprende-se na escola"), Futuras Aspirações e Objetivos (e.g., "É importante ir para a faculdade depois do ensino secundário") e Motivação Extrínseca (e.g., "Eu aprendo, mas apenas se o professor me der uma recompensa"). Já a subescala Envolvimento Escolar Psicológico debruça-se essencialmente sobre os sentimentos de identificação e pertencimento, e sobre 
a relação com os professores e pares, sendo constituída pelas subescalas Relação entre o Professor e o Aluno (e.g., "Os meus professores estão disponíveis para mim quando preciso deles"), Suporte Familiar para a Aprendizagem (e.g., "A minha família/pais está presente para mim quando preciso deles") e Apoio dos Pares para a Aprendizagem (e.g., "Os outros alunos aqui gostam de mim da forma como eu sou"). A versão portuguesa do instrumento apresenta valores de consistência interna aceitáveis, com alfa total de 0,84, variando entre 0,64 na subescala Aspirações Futuras e Objetivos, e 0,79 na subescala Relação entre os Professores e Alunos (Moreira et al., 2009).

Para avaliação das estratégias de otimização da aprendizagem foi utilizado o School Success Promoting Strategies Inventory (SPSI) (Moreira et al., 2013), instrumento desenvolvido por dois dos autores deste estudo. É um instrumento de autorrelato, constituído por 36 itens com tipo de resposta Likert, 1 = Discordo Totalmente; 2 = Discordo; 3 = Concordo; 4 = Concordo Totalmente. É constituído pelas subescalas Educação para a Carreira (e.g., "Os alunos têm oportunidade de realizar atividades que os preparam para o mundo profissional"), Aprendizagem Ativa (e.g., "Os professores dão oportunidades aos alunos que Ihes permitem desenvolver os seus projetos"), Tutoria (e.g., "Há uma pessoa responsável por me dar um acompanhamento personalizado"), Atividades Extracurriculares (e.g., "Há atividades interessantes em que os alunos se podem envolver depois das aulas"), Tecnologias Educativas (e.g., "Os alunos têm possibilidade de aprender com o apoio de computadores e material informático") e Aprendizagem Individualizada (e.g., "Os professores são flexíveis ao dar a matéria, consoante cada aluno"). O instrumento apresenta boa consistência interna, com valores de alfa de Cronbach entre 0,71 (Tecnologias Educativas) e 0,87 (Educação para a Carreira) (Moreira et al., 2014).

\section{Procedimentos}

A seleção da amostra teve em conta escolas pertencentes a um município envolvido na imple- mentação de um plano municipal de melhoria da escola. No protocolo estabelecido com o município constam todos os princípios orientadores sobre os quais se assentam os estudos desenvolvidos naquelas escolas. Dessa forma, estão asseguradas todas as questões éticas, bem como o anonimato dos participantes e a confidencialidade dos resultados.

A coleta dos dados aconteceu durante o mês de junho. As escolas atribuíram um número a cada uma das suas turmas e selecionaram aleatoriamente três turmas de cada ano letivo. Os alunos preencheram individualmente os dois questionários, disponibilizados online no site do Observatório da Melhoria e Eficácia da Escola, durante uma aula de 45 minutos. O preenchimento dos instrumentos na plataforma online foi supervisionado por um professor da escola.

Os dados recolhidos foram submetidos a diversas análises estatísticas, utilizando-se o Statistical Package for the Social Sciences (SPSS), versão 17.0.

\section{Resultados}

Na Tabela 1 estão representados os resultados obtidos (mínimo, máximo, média e desvio-padrão) nas dimensões e respectivos componentes, em cada uma das quatro escolas pesquisadas.

Ao analisar as diferenças entre as escolas (Tabela 2), no nível das dimensões e componentes avaliados, verifica-se que, quanto ao envolvimento escolar, existem diferenças estatisticamente significativas no nível da Motivação Extrínseca $(p=0,022)$ e Suporte Familiar para a Aprendizagem $(p=0,019)$ em relação aos demais níveis. Contudo, uma análise comparativa entre as escolas não aponta diferenças significativas no nível da Motivação Extrínseca, enquanto em relação ao Suporte Familiar para a Aprendizagem os demais alunos referem ter mais apoio dos pais para a aprendizagem que os da escola $C(p=0,019)$. Em relação às estratégias de Promoção do Sucesso Acadêmico, observam-se diferenças estatisticamente significativas na Educação para a Carreira $(p=0,000)$, Aprendizagem Ativa $(p=0,000)$, Tutoria $(p=0,000)$, Atividades Extracurriculares $(p=0,000)$, Aprendizagem Individualizada 
Tabela 1

Estatística descritiva das dimensões de envolvimento dos alunos com a escola e da percepção das estratégias de otimização da aprendizagem, por escola

\begin{tabular}{|c|c|c|c|c|c|}
\hline Escola & Dimensão & Mínimo & Máximo & Média & Desvio-Padrão \\
\hline \multirow{10}{*}{$\begin{array}{l}\text { Escola A } \\
(n=119)\end{array}$} & Envolvimento Cognitivo & 37 & 64 & 50,55 & 5,51 \\
\hline & Envolvimento Psicológico & 47 & 73 & 61,02 & 6,11 \\
\hline & Envolvimento Escolar & 89 & 134 & 111,57 & 9,05 \\
\hline & Educação para a Carreira & 11 & 28 & 21,28 & 3,85 \\
\hline & Aprendizagem Ativa & 13 & 28 & 21,39 & 3,14 \\
\hline & Tutoria & 5 & 14 & 7,75 & 2,71 \\
\hline & Atividades Extracurriculares & 6 & 16 & 11,28 & 2,61 \\
\hline & Tecnologias Educativas & 5 & 12 & 8,85 & 2,10 \\
\hline & Aprendizagem Individualizada & 4 & 11 & 6,72 & 1,53 \\
\hline & SPSI TOTAL & 59 & 98 & 77,28 & 9,28 \\
\hline \multirow{10}{*}{$\begin{array}{l}\text { Escola B } \\
(n=72)\end{array}$} & Envolvimento Cognitivo & 30 & 64 & 49,30 & 5,54 \\
\hline & Envolvimento Psicológico & 35 & 75 & 61,27 & 7,42 \\
\hline & Envolvimento Escolar & 79 & 131 & 110,57 & 9,48 \\
\hline & Educação para a Carreira & 12 & 26 & 21,27 & 2,68 \\
\hline & Aprendizagem Ativa & 11 & 28 & 21,57 & 3,18 \\
\hline & Tutoria & 5 & 18 & 11,19 & 4,47 \\
\hline & Atividades Extracurriculares & 4 & 16 & 10,78 & 2,54 \\
\hline & Tecnologias Educativas & 3 & 12 & 8,81 & 1,81 \\
\hline & Aprendizagem Individualizada & 3 & 12 & 8,20 & 2,25 \\
\hline & SPSI TOTAL & 39 & 102 & 81,84 & 12,32 \\
\hline \multirow{10}{*}{$\begin{array}{l}\text { Escola C } \\
(n=54)\end{array}$} & Envolvimento Cognitivo & 31 & 64 & 50,87 & 6,34 \\
\hline & Envolvimento Psicológico & 34 & 76 & 61,01 & 7,92 \\
\hline & Envolvimento Escolar & 78 & 139 & 111,89 & 12,55 \\
\hline & Educação para a Carreira & 15 & 27 & 21,90 & 2,92 \\
\hline & Aprendizagem Ativa & 15 & 28 & 22,96 & 3,00 \\
\hline & Tutoria & 5 & 20 & 11,74 & 4,52 \\
\hline & Atividades Extracurriculares & 5 & 16 & 12,63 & 2,72 \\
\hline & Tecnologias Educativas & 5 & 12 & 9,60 & 1,61 \\
\hline & Aprendizagem Individualizada & 3 & 12 & 8,57 & 2,03 \\
\hline & SPSI TOTAL & 59 & 115 & 87,41 & 12,54 \\
\hline \multirow{10}{*}{$\begin{array}{l}\text { Escola D } \\
(n=284)\end{array}$} & Envolvimento Cognitivo & 16 & 64 & 48,93 & 7,25 \\
\hline & Envolvimento Psicológico & 25 & 76 & 59,60 & 9,04 \\
\hline & Envolvimento Escolar & 41 & 137 & 108,53 & 14,62 \\
\hline & Educação para a Carreira & 7 & 28 & 19,47 & 3,99 \\
\hline & Aprendizagem Ativa & 7 & 28 & 20,44 & 3,87 \\
\hline & Tutoria & 5 & 20 & 9,58 & 4,05 \\
\hline & Atividades Extracurriculares & 4 & 16 & 10,24 & 3,05 \\
\hline & Tecnologias Educativas & 3 & 12 & 8,89 & 2,06 \\
\hline & Aprendizagem Individualizada & 3 & 12 & 7,50 & 2,57 \\
\hline & SPSI TOTAL & 32 & 113 & 76,12 & 14,25 \\
\hline
\end{tabular}

Nota: SPSI_Total: Soma de todos os itens do School Success Promoting Strategies Inventory.

$(p=0,000)$ e no total da escala $(p=0,000)$. Análises comparativas entre escolas demonstraram que, em relação à Educação para a Carreira, a escola C apresenta níveis superiores comparativamente às demais. Já quanto ao nível da Aprendizagem Ativa, a Escola D apresenta valores significativamente mais 192 baixos quando comparada com as outras. No nível da Tutoria, as escolas diferem de forma estatisticamente significativa entre si, exceto as escolas B e $C(p=0,452)$. Também no nível das Atividades Extracurriculares, Aprendizagem Individualizada e no nível do total das Estratégias, apenas as escolas com maiores índices se distinguem de forma estatisticamente significativa. 
Tabela 2

Médias (M), Desvio-Padrão (DP) e Teste de Diferença de Médias (Kruskal-Wallis) dos resultados, de todas as escolas, em todas as dimensões e componentes avaliados

\begin{tabular}{|c|c|c|c|c|c|c|c|c|c|c|c|}
\hline \multirow{2}{*}{ Dimensão } & \multicolumn{2}{|c|}{$\mathrm{A}(n=119)$} & \multicolumn{2}{|c|}{$\mathrm{B}(n=72)$} & \multicolumn{2}{|c|}{$C(n=54)$} & \multicolumn{2}{|c|}{$\mathrm{D}(n=284)$} & \multirow{2}{*}{$\mathrm{KW}$} & \multirow{2}{*}{$g l$} & \multirow{2}{*}{$p$} \\
\hline & $M$ & $D P$ & $M$ & $D P$ & $M$ & $D P$ & $M$ & $D P$ & & & \\
\hline Contr_Relev & 29,25 & 3,63 & 28,15 & 3,81 & 29,40 & 4,14 & 28,61 & 4,76 & 4.094 & 3 & 0.251 \\
\hline Asp_Futur & 17,58 & 2,08 & 17,32 & 2,20 & 17,40 & 2,08 & 16,96 & 3,05 & 1.245 & 3 & 0.742 \\
\hline Motiv_Extrín & 3,72 & 1,87 & 3,84 & 1,91 & 4,07 & 1,97 & 3,36 & 1,74 & 9.587 & 3 & $0.022^{*}$ \\
\hline Apoio_Prof & 27,48 & 4,08 & 28,05 & 4,48 & 27,89 & 4,57 & 26,60 & 5,54 & 6.416 & 3 & 0.093 \\
\hline Apoio_Família & 14,70 & 1,58 & 14,22 & 1,63 & 13,98 & 2,11 & 14,02 & 2,13 & 9.905 & 3 & $0.019^{*}$ \\
\hline Apoio_Pares & 18,83 & 2,05 & 19,00 & 3,09 & 19,15 & 2,85 & 18,99 & 3,06 & 2.496 & 3 & 0.476 \\
\hline Env_Cognit & 50,55 & 5,51 & 49,30 & 5,54 & 50,87 & 6,35 & 48,93 & 7,25 & 5.326 & 3 & 0.149 \\
\hline Env_Psicol & 61,02 & 6,11 & 61,27 & 7,42 & 61,02 & 7,92 & 59,60 & 9,04 & 2.246 & 3 & 0.523 \\
\hline Envolvimento Escolar & 111,57 & 9,05 & 110,58 & 9,48 & 111,89 & 12,55 & 108,53 & 14,62 & 2.347 & 3 & 0.504 \\
\hline Educação para a Carreira & 21,28 & 3,85 & 21,27 & 2,68 & 21,90 & 2,92 & 19,47 & 3,99 & 37.268 & 3 & $0.000^{*}$ \\
\hline Aprendizagem Ativa & 21,39 & 3,14 & 21,58 & 3,18 & 22,96 & 3,01 & 20,44 & 3,87 & 23.692 & 3 & $0.000^{*}$ \\
\hline Tutoria & 7,75 & 2,71 & 11,19 & 4,47 & 11,74 & 4,53 & 9,58 & 4,05 & 41.727 & 3 & $0.000^{*}$ \\
\hline Atividades Extracurriculares & 11,28 & 2,61 & 10,78 & 2,54 & 12,63 & 2,72 & 10,24 & 3,05 & 33.855 & 3 & $0.000^{*}$ \\
\hline Tecnologias Educativas & 8,85 & 2,11 & 8,81 & 1,81 & 9,60 & 1,61 & 8,89 & 2,06 & 6.716 & 3 & 0.103 \\
\hline Aprendizagem Individualizada & 6,73 & 1,53 & 8,21 & 2,25 & 8,57 & 2,03 & 7,50 & 2,57 & 35.940 & 3 & $0.000^{*}$ \\
\hline SPSI_TOTAL & 77,28 & 9,28 & 81,84 & 12,33 & 87,41 & 12,55 & 76,12 & 14,25 & 37.522 & 3 & $0.000^{*}$ \\
\hline
\end{tabular}

Nota: ${ }^{*} p \leq 0,05$.

Contr_Relev: Controle e Relevância do Trabalho da Escola; Asp_Futur: Aspirações e Objetivos Futuros; Motiv_Extrín: Motivação Extrínseca; Apoio_Prof: Apoio dos professores para a aprendizagem; Apoio_Família: Suporte familiar para a aprendizagem; Apoio_Pares: Apoio dos pares para a aprendizagem; Env_Cognit: Envolvimento Cognitivo; Env_Psicol: Envolvimento Psicológico; SPSI_Total: Soma de todos oS itens do School Success Promoting Strategies Inventory; KW: teste Kruskal-Wallis; gl: graus de liberdade.

Existe uma correlação estatisticamente significativa ( $p=0,000$ ) entre o Envolvimento Escolar Total dos alunos e o Total das Estratégias de Promoção do Sucesso Acadêmico (Students' Perceptions of School Success Inventory [SPSI]) (Moreira et al., 2014). Em relação à subescala Controle e Relevância do Trabalho da Escola, ela se encontra correlacionada negativamente com a Motivação Extrínseca $(r=-0,057, p=0,191)$, e positivamente correlacionada com a Tutoria $(r=0,066, p=0,126)$.

Quanto à subescala Aspirações e Objetivos Futuros, ela se correlaciona de modo estatisticamente significativo $(p=0,000)$ com todas as subescalas, com exceção da Tutoria. A Relação Professor/Aluno está correlacionada estatisticamente $(p=0,000)$ com todas as dimensões, com exceção da Motivação Extrínseca $(r=-0,018, p=0,687)$. 0 Suporte Familiar para a Aprendizagem está estatisticamente correlacionado com todas as dimensões avaliadas $(p=0,000)$, com exceção para a Tutoria $(r=-0,011, p=0,794)$. O Apoio dos Pares para a Aprendizagem encontra-se correlacionado, de modo estatisticamente significativo, com todas as dimensões $(p=0,000)$, exceto com a Motivação Extrínseca $(r=0,015, p=0,733)$.

Em relação à Educação para a Carreira, ela se encontra correlacionada significativamente com todas as dimensões: Controle e Relevância do Trabalho da Escola ( $r=0,329, p=0,000)$, Aspirações e Objetivos Futuros $(r=0,259, p=0,000)$, Motivação Extrínseca $(r=0,094, p=0,029)$ e Relação Professor/ Aluno $(r=0,331, p=0,000)$.

A Aprendizagem Ativa encontra-se correlacionada a um nível estatisticamente significativo com todas as dimensões: Controle e Relevância do Trabalho da Escola $(r=0,428 p=0,000)$, Aspirações e Objetivos Futuros $(r=0,359, p=0,000)$ e Relação Professor/Aluno $(r=0,391, p=0,000)$, mas não está correlacionada com a Motivação Extrínseca $(r=0,011, p=0,802)$. Quanto à Tutoria, os resultados mostram que está estatisticamente correlacionada com a Motivação Extrínseca $(r=0,242$, $p=0,000)$ e com as Relação Professor/Aluno $(r=0,160, p=0,000)$.

A dimensão Atividades Extracurriculares está estatisticamente correlacionada com o Controle e 
Relevância do Trabalho da Escola $(r=0,340, p=0,000)$, com as Aspirações e Objetivos Futuros $(r=0,234$, $p=0,000)$ e com as Relação Professor/Aluno $(r=0,241$, $p=0,000$ ).

A dimensão Tecnologias Educativas encontra-se estatisticamente correlacionada com todas as dimensões Controle e Relevância do Trabalho da Escola ( $r=0,335, p=0,000)$, Aspirações e Objetivos Futuras $(r=0,298, p=0,000)$ e Relação Professor/Aluno $(r=0,221, p=0,000)$. Tendencialmente verifica-se que a percepção dos alunos em relação às estratégias de promoção do sucesso escolar está mais correlacionada com os indicadores de envolvimento dos estudantes com a escola, como Controle e Relevância do Trabalho da Escola $(r=0,42)$, Relação Professor/Aluno $(r=0,40)$, e menos correlacionada com indicadores como Motivação Extrínseca $(r=0,12)$ ou Suporte Familiar para a Aprendizagem $(r=0,18)$. Esses resultados estão representados na Tabela 3.

Analisou-se também o envolvimento dos alunos com a escola e a percepção acerca das estratégias de promoção do sucesso escolar por ciclo de ensino (Tabela 4). Verifica-se que existem diferenças estatisticamente significativas no nível do Controle e Relevância do Trabalho da Escola $(p=0,004)$, das Aspirações e Objetivos Futuros $(p=0,048)$, da Relação Professor/Aluno $(p=0,002)$, do Envolvimento Escolar Cognitivo $(p=0,001)$ e do Envolvimento Escolar Total $(p=0,004)$. As dimensões relacionadas com as estratégias de otimização da aprendizagem diferenciam-se, de forma estatisticamente significativa, nos níveis da Educação para a Carreira $(p=0,001)$, Aprendizagem Ativa $(p=0,000)$, Tutoria $(p=0,046)$, Atividades Extracurriculares $(p=0,000)$ e Aprendizagem Individualizada $(p=0,000)$, além de se diferenciarem no seu total $(p=0,000)$. Neste estudo, os alunos do $2^{\circ}$ Ciclo tendem a apresentar maior envolvimento com a escola e percebem mais estratégias de promoção do sucesso escolar, quando comparados com os alunos do $3^{\circ} \mathrm{Ciclo}$.

\section{Discussão}

O objetivo deste estudo foi avaliar a relação entre a percepção dos alunos acerca das estratégias de promoção do sucesso escolar postas em prática

Tabela 3

Correlações entre dimensões e componentes do Envolvimento Escolar e das Estratégias de Promoção do Sucesso Acadêmico

\begin{tabular}{|c|c|c|c|c|c|c|c|c|c|c|c|c|c|c|c|c|}
\hline & Dimensão & 1 & 2 & 3 & 4 & 5 & 6 & 7 & 8 & 9 & 10 & 11 & 12 & 13 & 14 & 15 \\
\hline & \multicolumn{16}{|l|}{ 1. Contr_Relev } \\
\hline & 2. Asp_Futur & $0,73^{* *}$ & & & & & & & & & & & & & & \\
\hline & 3. Motiv_Extrín & $-0,06$ & $-0,17^{\star *}$ & & & & & & & & & & & & & \\
\hline & 4. Rel_Prof_A & $0,46^{* *}$ & $0,38^{* *}$ & $-0,02$ & & & & & & & & & & & & \\
\hline & 5. Apoio_Fam & $0,39^{* *}$ & $0,43^{* *}$ & $-0,17^{\star *}$ & $0,42^{* *}$ & & & & & & & & & & & \\
\hline & 6. Apoio_Pares & $0,32^{* *}$ & $0,30^{* *}$ & 0,01 & $0,55^{* *}$ & $0,45^{* *}$ & & & & & & & & & & \\
\hline & 7. Env_Cognit & $0,94^{* *}$ & $0,84^{* *}$ & $0,17^{* *}$ & $0,45^{* *}$ & $0,38^{* *}$ & $0,33^{* *}$ & & & & & & & & & \\
\hline & 8. Env_Psicol & $0,49^{* *}$ & $0,44^{* *}$ & $-0,05$ & $0,91^{* *}$ & $0,66^{* *}$ & $0,80^{* *}$ & $0,49^{* *}$ & & & & & & & & \\
\hline & 9. Env_Escolar & $0,80^{* *}$ & $0,71^{* *}$ & 0,06 & $0,82^{* *}$ & $0,62^{* *}$ & $0,68^{* *}$ & $0,83^{* *}$ & $0,89^{* *}$ & & & & & & & \\
\hline & 10. Edu_Carreira & $0,33^{* *}$ & $0,26^{* *}$ & $0,09^{*}$ & $0,33^{* *}$ & $0,18^{* *}$ & $0,17^{* *}$ & $0,35^{* *}$ & $0,31^{* *}$ & $0,38^{* *}$ & & & & & & \\
\hline & 11. Apren_Ativa & $0,43^{* *}$ & $0,36^{* *}$ & 0,01 & $0,39^{* *}$ & $0,19^{* *}$ & $0,22^{* *}$ & $0,43^{* *}$ & $0,36^{* *}$ & $0,46^{* *}$ & $0,65^{* *}$ & & & & & \\
\hline & 12. Tutoria & 0,07 & $-0,01$ & $0,24^{* *}$ & $0,16^{* *}$ & $-0,01$ & $0,16^{* *}$ & $0,10^{*}$ & $0,15^{* *}$ & $0,15^{* *}$ & $0,26^{* *}$ & $0,26^{\star *}$ & & & & \\
\hline & 13. Ativ_Extra & $0,34^{* *}$ & $0,23^{* *}$ & 0,03 & $0,24^{* *}$ & $0,14^{* \star}$ & $0,13^{* *}$ & $0,33^{* *}$ & $0,23^{* *}$ & $0,32^{* *}$ & $0,43^{* *}$ & $0,50^{* *}$ & $0,30^{* *}$ & & & \\
\hline & 14. Tecnol_Edu & $0,33^{* *}$ & $0,30^{* *}$ & $-0,05$ & $0,22^{* *}$ & $0,18^{* *}$ & $0,15^{* *}$ & $0,33^{* *}$ & $0,23^{* *}$ & $0,32^{* *}$ & $0,24^{* *}$ & $0,45^{* *}$ & $0,14^{* *}$ & $0,51^{* *}$ & & \\
\hline & 15. Aprend_Indi & $0,36^{* *}$ & $0,22^{* *}$ & $0,10^{*}$ & $0,37^{* *}$ & $0,12^{* *}$ & $0,17^{\star *}$ & $0,36^{* *}$ & $0,32^{* *}$ & $0,39^{* *}$ & $0,36^{* *}$ & $0,50^{* *}$ & $0,43^{* *}$ & $0,48^{* *}$ & $0,47^{* *}$ & \\
\hline \multirow{4}{*}{ 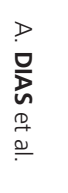 } & 16. SPSI_TOTAL & $0,42^{* *}$ & $0,30^{* *}$ & $0,12^{* *}$ & $0,40^{* *}$ & $0,18^{* *}$ & $0,24^{* *}$ & $0,43^{* *}$ & $0,38^{* *}$ & $0,46^{* *}$ & $0,74^{* *}$ & $0,81^{* *}$ & $0,62^{* *}$ & $0,74^{* *}$ & $0,58^{* *}$ & $0,73^{* *}$ \\
\hline & Comelaçau & ant & (aty & IIVd & $p=$ & $\mathrm{Col}$ & J Meldç & Estatis & dment & . & Pr & $p \leq 0,01$. & & & & \\
\hline & Contr_Relev: Contro & e Relevân & cia do T & Trabalho & da Escola & ; Asp_Fu & utur: Aspi & irações e & Objetiv & os Futurc & s; Motiv & Extrín: N & Motivaçã & Extrínse & ca; Relac & EProf_A: \\
\hline & Relação entre profes & res e alun & רos; Apoi & io_Fam: $S$ & Suporte $f$ & amiliar p & ara a apr & rendizage & em; Apo & io_Pares & Apoio d & los pares & para a a & prendiza & gem; Env & I_Cognit: \\
\hline 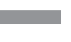 & Envolvimento Cogni & o; Env_Ps & sicol: Envc & olvimentc & o Psicolós & gico; Env & V_Escolar: & : Envolvir & mento $\mathrm{E}$ & scolar; E & du_Carre & ira: Educ & ação $p$ & a a Carre & eira; Apr & en_Ativa: \\
\hline & Aprendizabem Ativa & iv_Extra: & : Atividad & des Extra- & Curricular & res; Tecn & ol_Edu: T & To to & $f_{0} 5 \mathrm{~d}$ & tivac. & rend Ind & $10 m p^{2}$ & & & & \\
\hline & & & & & & & 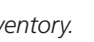 & & & & & & & & & \\
\hline
\end{tabular}


Tabela 4

Média (M), Desvio-Padrão (DP) e teste de diferença de médias (t Student) para as diferentes dimensões e componentes, entre o $2^{\circ}$ e $3^{\circ}$ Ciclos de todas as escolas

\begin{tabular}{|c|c|c|c|c|c|c|}
\hline \multirow{2}{*}{ Dimensões } & \multicolumn{2}{|c|}{$2^{\circ} \mathrm{Ciclo}(n=216)$} & \multicolumn{2}{|c|}{$3^{\circ}$ Ciclo $(n=313)$} & \multirow{2}{*}{$t$} & \multirow{2}{*}{$p$} \\
\hline & M & $D P$ & $M$ & $D P$ & & \\
\hline Contr_Relev & 29,44 & 4,04 & 28,33 & 4,48 & 2,901 & $0.004^{*}$ \\
\hline Asp_Futur & 17,48 & 2,55 & 17,01 & 2,73 & 1,986 & $0.048^{*}$ \\
\hline Motiv_Extrín & 3,74 & 1,87 & 3,46 & 1,80 & 1,714 & 0.087 \\
\hline Relac_Prof_Alun & 27,93 & 4,45 & 26,54 & 5,33 & 3,154 & $0.002^{*}$ \\
\hline Apoio_Família & 14,29 & 1,89 & 14,12 & 2,03 & 1,013 & 0.312 \\
\hline Apoio_Pares & 18,85 & 2,94 & 19,04 & 2,77 & 0,760 & 0.447 \\
\hline Env_Cognit & 50,66 & 6,07 & 48,81 & 6,84 & 3,197 & $0.001^{*}$ \\
\hline Env_Psicol & 61,08 & 7,81 & 59,70 & 8,33 & 1,916 & 0.056 \\
\hline Envolvimento Escolar & 111,73 & 12,54 & 108,51 & 12,75 & 2,880 & $0.004^{*}$ \\
\hline Educação para a Carreira & 21,01 & 3,35 & 19,93 & 4,07 & 3,232 & $0.001^{*}$ \\
\hline Aprendizagem Ativa & 21,72 & 3,26 & 20,59 & 3,78 & 3,591 & $0.000^{*}$ \\
\hline Tutoria & 10,03 & 4,34 & 9,31 & 3,90 & 2,003 & $0.046^{*}$ \\
\hline Atividades Extracurriculares & 11,47 & 2,87 & 10,30 & 2,91 & 4,573 & $0.000^{*}$ \\
\hline Tecnologias Educativas & 8,98 & 1,97 & 8,91 & 2,03 & 0,400 & 0.689 \\
\hline Aprendizagem Individualizada & 8,26 & 2,36 & 7,03 & 2,21 & 6,097 & $0.000^{*}$ \\
\hline SPSI_TOTAL & 81,48 & 12,96 & 76,07 & 13,13 & 4,682 & $0.000^{*}$ \\
\hline
\end{tabular}

Nota: * $p \leq 0,05$.

Contr_Relev: Controle e Relevância do Trabalho da Escola; Asp_Futur: Aspirações e Objetivos Futuros; Motiv_Extrín: Motivação Extrínseca; Relac_Prof_Alun: Relação entre professores e alunos; Apoio_Família: Suporte familiar para a aprendizagem; Apoio_Pares: Apoio dos pares para a aprendizagem; Env_Cognit: Envolvimento Cognitivo; Env_Psicol: Envolvimento Psicológico; SPSI_Total: Soma de todos os itens do School Success Promoting Strategies Inventory.

pelas escolas e o envolvimento dos alunos com a escola. Os resultados demonstram a existência de correlações estatisticamente significativas entre as várias dimensões de envolvimento escolar e a percepção dos alunos acerca do grau em que as escolas fomentam estratégias de promoção do sucesso educativo. A seguir, serão discutidos os vários resultados encontrados.

\section{Relação entre a percepção dos alunos acerca da adoção de estratégias de promoção do sucesso acadêmico por parte das escolas e o envolvimento dos alunos com a escola}

Os resultados deste estudo demonstram correlações positivas e estatisticamente significativas entre todas as dimensões de envolvimento dos alunos com a escola e a percepção destes acerca da promoção de estratégias de sucesso escolar. Os resultados sugerem que uma percepção mais positiva acerca da adoção de estratégias de promoção do sucesso educativo é encontrada em alunos com níveis mais elevados de envolvimento com a escola.

Esses resultados são congruentes com outros estudos, principalmente os que sugerem que as percepções positivas dos alunos acerca dos esforços de melhoria da escola influenciam o nível de envolvimento deles com a escola (Cavendish, 2013; Lee \& Burkam, 2003; Van Ryzin, 2011).

Apesar de estudos anteriores sugerirem que as características da escola (sobretudo as características organizacionais) têm o potencial de provocar um efeito positivo no envolvimento dos alunos, os resultados do presente estudo não permitem identificar relações de causa-efeito. De fato, não é possível inferir se são as percepções acerca dos esforços de melhoria que têm impacto no envolvimento dos alunos com a escola, ou se os níveis de envolvimento dos alunos com a escola é que têm um impacto em suas percepções acerca dos esforços de melhoria. O caráter dinâmico entre as percepções dos alunos e as características contextuais (Van Ryzin, 2011) sugere que essas influências são bidireccionais e dinâmicas. 
Apesar de o estabelecimento de relações de causa e efeito requerer estudos com outras metodologias (nomeadamente experimentais e longitudinais), os resultados deste estudo contribuem para uma melhor compreensão das associações entre as percepções dos alunos acerca das características contextuais e o envolvimento com a escola. Os índices das correlações encontradas, principalmente no nível dos indicadores globais de percepção acerca das estratégias de promoção do sucesso educativo e de envolvimento com a escola, que se situam em valores superiores a 0,4 , sugerem associações médias em termos de magnitude, o que significa que os dois fenômenos covariam fortemente.

\section{Diferenças entre escolas}

Os resultados deste estudo relevam a existência de diferenças entre as quatro escolas, principalmente quanto às percepções dos alunos acerca das estratégias de promoção do sucesso educativo. Curiosamente, essas diferenças não foram detectadas na maioria dos indicadores de envolvimento com a escola, com exceção da Motivação Extrínseca e do Apoio dos Professores para a Aprendizagem. Esses resultados sugerem que as diferenças encontradas entre as escolas se situam majoritariamente no nível das dimensões contextuais, mais do que no nível das dimensões individuais. Apesar de as duas dimensões covariarem, como demonstrado pelas correlações, as diferenças entre escolas encontram-se sobretudo no grau em que os alunos percepcionam os esforços de melhoria implementados por cada escola.

De forma consistente com as evidências existentes, os resultados deste estudo confirmam a forte associação entre questões contextuais (estratégias promovidas pelas escolas) e individuais (percepções e envolvimento com a escola). Essas dinâmicas têm sido extensamente explicadas por modelos interacionistas meio-indivíduo, dentre os quais o modelo bioecológico do desenvolvimento humano é um exemplo (Bronfenbrenner, 2009). Por um lado, as características de um contexto ou mesossistema (as características da escola) influenciam as dimensões envolvimento dos alunos com a escola). Por outro lado, as características do microssistema também têm impacto nas características dos contextos, seja em termos da sua modulação, seja em termos das percepções acerca das mesmas (Bronfenbrenner, 2009).

\section{Diferenças entre ciclos de estudos}

Ao se analisarem os ciclos, verifica-se que em todas as escolas do Município de Vila Nova de Famalicão, com exceção da escola A, os alunos do $2^{\circ}$ Ciclo referem níveis mais desejáveis de estratégias de otimização da aprendizagem, quando comparados com alunos do $3^{\circ} \mathrm{Ciclo}$. Além disso, apresentam valores mais elevados de Envolvimento Escolar (Cognitivo e Psicológico), quando comparados aos do terceiro ciclo.

Tais dados vão ao encontro dos resultados obtidos em outros estudos, segundo os quais os alunos tendem a apresentar uma diminuição do interesse e importância em relação às tarefas escolares ao longo do seu percurso acadêmico (Fredericks, Blumenfeld, \& Paris, 2004; Fredericks \& Eccles, 2002). Essa variação pode ser explicada à luz das mudanças desenvolvimentais dos alunos, isto é, crianças/jovens mais novos tendem a ser mais otimistas em relação às suas competências (Nicholls \& Miller, 1984) do que crianças/jovens mais velhos, que tendem a ter uma visão mais crítica acerca das suas capacidades (Fredericks \& Eccles, 2002). Além disso, dimensões disposicionais e relativamente estáveis ao longo do tempo, como a personalidade, exercem uma influência significativa em múltiplos resultados acadêmicos, incluindo o desempenho escolar e o envolvimento com a escola (Moreira et al., 2012d).

Paralelamente, o tipo de avaliação utilizada pelos professores também se altera, passando de critérios centrados no desenvolvimento da mestria, característicos do ensino básico, para uma avaliação mais comparativa e normativa em ciclos de estudos mais avançados, promovendo a competitividade e a comparação de competências entre os alunos (Fredericks \& Eccles, 2002). 


\section{Implicações dos resultados deste estudo para os esforços de melhoria da escola}

Os esforços de melhoria da escola têm como principal objetivo a implementação de estratégias que favoreçam o desenvolvimento de indicadores mais adaptativos de trajetórias acadêmicas. Os indicadores de trajetórias acadêmicas variam desde indicadores de resultados (como notas escolares) até indicadores de processo (como envolvimento com a escola). As contribuições da Psicologia para a melhoria e eficácia da escola incluem a compreensão das dinâmicas entre as dimensões contextuais e individuais envolvidas nas trajetórias acadêmicas.

Dessa forma, e atendendo a que a principal função da escola é proporcionar as condições adequadas para o desenvolvimento dos alunos, de acordo com as evidências disponíveis, as escolas precisam estar conscientes da importância de moldar suas práticas de modo a proporcionar efeitos o mais positivos possível para aquele desenvolvimento (Moreira et al., 2015). Estudos demonstram que alunos de escolas com características diferentes tendem a apresentar valores também diferentes no nível de várias dimensões (incluindo dimensões dos modelos motivacionais e dos modelos de persistência educacional) (Moreira et al., 2012a). Assim, a adoção de estratégias de promoção do sucesso educativo por parte das escolas é de grande relevância, uma vez que, como é sugerido também por este estudo, elas interagem com dimensões cognitivas, como as percepções dos alunos, e com dimensões emocionais e comportamentais, na organização dos processos psicológicos determinantes do sistema comportamental do aluno. Por outras palavras, se o envolvimento dos alunos com a escola é uma dimensão iminentemente subjetiva de cada qual, ele resulta de dinâmicas complexas e está associado a dimensões passíveis de intervenção. As fortes associações positivas encontradas neste estudo entre a percepção dos alunos acerca dos esforços de melhoria promovidos pela escola e o envolvimento desses estudantes com os estudos, apontam a necessidade de as escolas desenvolverem programas intencionais de melhoria.

\section{Referências}

Abbott Chapman, J., Martin, K., Ollington, N., Venn, A., Dwyer, T., \& Gall, S. (2013). The longitudinal association of childhood school engagement with adult educational and occupational achievement: Findings from an Australian national study. British Educational Research Journal, 40(1), 102-120.

Adelman, H. S., \& Taylor, L. (1993). Learning problems and learning disabilities: Moving forward. Pacific Grove, CA: Brooks/Cole.

Ainscow, M. (2010). Achieving excellence and equity: Reflections on the development of practices in one local district over 10 years. School Effectiveness and School Improvement, 21(1), 75-92.

Appleton, J., Christenson, S. L., Kim, D., \& Reschly, A. (2006). Measuring cognitive and psychological engagement: Validation of the student engagement instrument. Journal of School Psychology, 44(5), 427-445.

Bell, B. S., \& Kozlowski, S. W. (2008). Active learning: Effects of core training design elements on selfregulatory processes, learning, and adaptability. Journal of Applied Psychology, 93(2), 296-316.

Bronfenbrenner, U. (2009). The ecology of human development: Experiments by nature and design. Cambridge, MA: Harvard University Press.

Bryk, A., Easton, J., Kerbeow, D., Rollow, S., \& Sebring, P. (1994). The state of Chicago school reform. Phi Delta Kappan, 76(1), 74-81.

Cavendish, W. (2013). Student perceptions of school efforts to facilitate student involvement, school commitment, self-determination, and high school graduation. Social Psychology of Education, 16(2), 257-275. http://dx.doi.org/10.1007/s11218-013-9212-z

Costa, J. A., Sousa, L., \& Neto-Mendes, A. (2000). Gestão pedagógica e lideranças intermédias no TEIP do Esteiro. In A. M. Bettencourt (Org.), Territórios educativos de intervenção prioritária: construção ecológica da acção educativa (pp.83-104). Lisboa: Instituto de Inovação Educacional.

Creemers, B., \& Reezigt, G. (2005). Linking school effectiveness and school improvement: The background and outline of the project. School Effectiveness \& School Improvement, 16(4), 359-371.

DeKalb, J. (1999). Student truancy. ER/C Digest, (125), 99-101.

Epstein, J. L., \& Sheldon, S. B. (2002). Present and accounted for: Improving student attendance through family and community involvement. The Journal of Educational Research, 95(5), 308-318.

Feldman, A. F., \& Matjasko, J. L. (2005). The role of schoolbased extra-curricular activities in adolescent development: A comprehensive review and future directions. Review of Educational Research, 75(2), 159-210. 
Ferreira, J. A., Santos, E., Fonseca, A. C., \& Haase, R. F. (2007). Early predictors of career development: A tenyear follow-up study. Journal of Vocational Behavior, 70(1), 61-77.

Fredericks, J. A., Blumenfeld, P. C., \& Paris, A. H. (2004). School engagement: Potential of the concept, state of the evidence. Review of Educational Research, 74(1), 59-109.

Fredricks, J. A., \& Eccles, J. S. (2002). Children's competence and value beliefs from childhood through adolescence: Growth trajectories in two male-sex-typed domains. Developmental Psychology, 38(4), 519-533.

Fredricks, J., McColskey, W., Meli, J., Montrosse, B., Mordica, J., \& Mooney, K. (2011). Measuring student engagement in upper elementary through high school: A description of 21 instruments. Washington, DC: Institute of Education Sciences. Retrieved September 30, 2013, from http://ies.ed.gov/ncee/ edlabs

Gilman, R., Meyers, J., \& Perez, L. (2004). Structured extracurricular activities among adolescents: Findings and implications for school psychologists. Psychology in the Schools, 41(1), 3-41.

Gonzales, R., Richards, K., \& Seeley, K. (2002). Youth out of school: Linking absence to delinquency. Denver: Colorado Foundation for Families and Children. Retrieved November 12, 2006, from http://www. truancyprevention.org/documents/DonnerComplete904.pdf

Gullatt, D., \& Lemoine, D. (1997). Truancy: What's a principal to do? American Secondary Education, 26(1), 7-12.

Hartley, J. (2007). Teaching, learning and new technology: A review for teachers. British Journal of Educational Technology, 38(1), 42-62.

Henry, K. L., Knight, K. E., \& Thornberry, T. P. (2012). School disengagement as a predictor of dropout, delinquency, and problem substance use during adolescence and early adulthood. Journal of Youth and Adolescence, 41(2), 156-166.

Hofman, R. H., Dijkstra, N. J., \& Hofman, W. H. A. (2005). School self-evaluation instruments: An assessment framework. International Journal of Leadership in Education, 8(3), 253-272.

Imel, S. (1999). Using technologies effectively in adult and vocational education. Literacy Practitioner, 5(2). 12-14.

Lee, V. E., \& Burkam, D. T. (2003). Dropping out of high school: The role of school organization and structure. American Educational Research Journal, 40(2), 353-393.

McKeown, R. (2002). Progress has been made in education for sustainable development? Applied Environmental Education and Communication, 1(1), 21-41.

Moreira, P. A. S., Vaz, F. M., Dias, P., \& Petracchi, P. (2009). Psychometric properties of the Portuguese version of the school engagement instrument. Canadian Journal of School Psychology, 24(4), 303-317. http://dx.doi. org/10.1177/0829573509346680

Moreira, P. A. S., Dias, P. C., Vaz, F. M., Rocha, C., Monteiro, J., \& Vaz, J. M. (2012a). Escolas secundárias Portuguesas com melhores e piores resultados académicos: dimensões do aluno, da família e da escola. Revista de Psicologia da Criança e do Adolescente, 3(1), 81-122.

Moreira, P. A. S., Dias, P., Pettrachi, P., \& Vaz, F. M. (2012b). Características psicométricas do Questionário de Envolvimento entre Pais e Professores. Revista de Psicologia da Criança e do Adolescente, 3(1), 123-135.

Moreira, P. A. S., Dias, P., Vaz, F. M., Rocha, C., \& Freitas, S. (2012C). Preditores de desempenho académico em alunos Portugueses do $12^{\circ}$ ano: comparação entre escolas com diferentes perfis. In J. M. Matos, J. Verdasca, M. Matos, M. E. Costa, M. E. Ferrão, \& P. A.S. Moreira (Coords.), Promoção do sucesso educativo: Projectos de pesquisa (pp.189-233). Lisboa: Fundação Calouste Gulbenkian.

Moreira, P. A. S., Oliveira, J. T., Cloninger, K. M., Azevedo, C., Sousa, A., Castro, J., \& Cloninger, C. R. (2012d). The psychometrics and validity of the junior temperament and character inventory in Portuguese adolescents. Comprehensive Psychiatry, 53(8), 1227-1236. http://dx.doi.org/10.1016/j.comppsych. 2012.04.014

Moreira, P. A. S, Dias, P., Vaz, F. M., \& Vaz, J. M. (2013). Predictors of academic performance and school engagement: Integrating persistence, motivation and study skills perspectives using person-centered and variable-centered approaches. Learning and Individual Differences, 24(2), 117-125. http://dx.doi.org/10.10 16/j.lindif.2012.10.016

Moreira, P. A. S., Oliveira, J. T., Dias, P., Vaz, F. M., \& TorresOliveira, I. (2014). The students' perceptions of school Success Promoting Strategies Inventory (SPSI): Development and validity evidence based studies. The Spanish Journal of Psychology, 17, E61. http://dx.doi. org/10.1017/sjp.2014.62

Moreira, P. A., Cloninger, C. R., Dinis, L., Sá, L., Oliveira, J. T., Dias, A., \& Oliveira, J. (2015). Personality and well-being in adolescents. Frontiers in Psychology, 5, 1494. http://dx.doi.org/10.3389/fpsyg.2014.01494

Motti-Stefanidi, F., \& Masten, A. S. (2013). School success and school engagement of immigrant children and adolescents. European Psychologist, 18(2), 126-135.

Nicholls, J., \& Miller, A. T. (1984). Development and its discontents: The differentiation of the concept of ability. In J. Nicholls (Ed.), Advances in motivation and achievement: The development of achievement motivation (Vol. 3., pp.185-218). Greenwich, CT: JAI Press.

Reynolds, D., Harris, A., Clarke, P., Harris, B., \& James, S. (2006). Challenging the challenged: Developing an 
improvement programme for school facing exceptionally challenging circumstances. School Effectiveness \& School Improvement, 17(4), 425-439.

Schildkamp, K., Visscher, A. J., \& Luyten, H. (2009). The effects of the use of a school self-evaluation instrument. School effectiveness and school improvement, 20(1), 69-88.

Stainback, W., \& Stainback, S. (1992). Controversial issues confronting special education. Boston: Allyn and Bacon.

Stone, J. R. (2004). Career and technical education: Increasing school engagement. In J. Smink \& F. P. Schargel (Eds.), Helping students graduate: A strategic approach to dropout prevention (pp.177-184). Larchmont, NY: Eye on Education.

Stone, J. R., \& Aliaga, O. A. (2002). Career and technical education, career pathways and work-based learning: Changes in participation 1997-1999. Saint Paul, MN: National Research Center for Career and Technical Education.

Sun, H., Creemers, B. P. M., \& de Jong, R. (2007). Contextual factors and effective school improvement. School Effectiveness \& School Improvement, 18(1), 93-122.

Switzer, D. (2004). Individualized instruction. In J. Smink, \& F. P. Schargel (Eds.), Helping students graduate: A strategic approach to dropout prevention (pp.225-233). Larchmont, NY: Eye on Education.

Taraban, R., Box, C., Myers, R., Pollard, R., \& Bowen, C. $W$. (2007). Effects of active-learning experiences on achievement, attitudes, and behaviors in High School Biology. Journal of Research in Science Teaching, 44(7), 960-979.

Taylor, L., \& Adelman, H. S. (1999). Personalizing classroom instruction to account for motivational and developmental differences. Reading and Writing Quarterly, 15(4), 255-276.

Tierney, J. P., Grossman, J. B., \& Resch, N. L. (1995). Making a difference: An impact study of Big/Brothers/Big
Sisters (Executive Summary). Philadelphia: Public/Private Ventures.

Totura, C. M. W., Karver, M. S., \& Gesten, E. L. (2013). Psychological distress and student engagement as mediators of the relationship between peer victimization and achievement in middle school youth. Journal of Youth and Adolescence, 98(1), 1-13. http://dx.doi.org/ 10.1007/s10964-013-9918-4

United Nations Educational, Scientific, Cultural Organization. (2005). Promotion of a global partnership for the UN decade of education for sustainable development: The International implementation scheme in brief. Paris: Unesco.

United Nations Educational, Scientific, Cultural Organization. (2008). Inclusive education: The way of the future. Geneva: International Conference on Education.

Van Ryzin, M. J. (2011). Protective factors at school: Reciprocal effects among adolescents' perceptions of the school environment, engagement in learning, and hope. Journal of Youth and Adolescence, 40(12), 1568-1580. http://dx.doi.org/10.1007/s10964-011-9 637-7

Vare, P., \& Scott, W. A. H. (2007). Learning for a change: Exploring the relationship between education and sustainable development. Journal of Education for Sustainable Development, 1(2), 191-198.

Wagstaff, M., Combs, L., \& Jarvis, B. (2000). Solving high school attendance problems: A case study. Journal of At-Risk Issues, 7(1), 21-30.

Wesley, T. (2004). Educational technology: Why and how it counts for students at risk. In J. Smink \& F. P. Schargel (Eds.), Helping students graduate: A strategic approach to dropout prevention (pp.211-223). Larchmont, NY: Eye on Education.

Yoder, J. D., \& Hochevar, C. M. (2005). Encouraging active learning can improve students' performance on examinations. Teaching of Psychology, 32(2), 91-95.

Recebido: maio 20, 2013

Versão final: janeiro 23, 2014

Aprovado: outubro 2, 2014 
